\title{
Immunoaffinity Chromatography
}

National Cancer Institute

\section{Source}

National Cancer Institute. Immunoaffinity Chromatography. NCI Thesaurus. Code C75519.

Affinity chromatography using monoclonal or polyclonal antibodies, or (less commonly) antigens, as the immobilized binding phase. 